\title{
Rabies: Repetition of an Old Tragic Story in Southeastern Iran
}

\author{
Jalil Nejati (iD) ${ }^{1}$, Mehdi Zanganeh Baygi (iD) ${ }^{2,}$ and Said Salemroudi ${ }^{3}$ \\ ${ }^{1}$ Health Promotion Research Center, Zahedan University of Medical Sciences, Zahedan, Iran \\ ${ }^{2}$ Department of Public Health, Health Promotion Research Center, School of Health, Zahedan University of Medical Sciences, Zahedan, Iran \\ ${ }^{3}$ Zahedan Health Center, Zahedan University of Medical Sciences, Zahedan, Iran \\ "Corresponding author: Department of Public Health, Health Promotion Research Center, School of Health, Zahedan University of Medical Sciences, Zahedan, Iran. Email: \\ dr.mzanganeh@hotmail.com
}

Received 2021 November 19; Revised 2021 December 27; Accepted 2022 January 07.

Keywords: Rabies, Iran, Zahedan

\section{Dear Editor,}

Rabies is one of the most important zoonotic diseases in the world. This viral infectious agent has $100 \%$ mortality in humans and animals (1) so that all patients with clinical symptoms finally die. It is estimated that annually rabies causes more than 59,000 deaths worldwide (2). Every nine minutes, one person dies due to this disease globally, and $30-50 \%$ of the victims are children under 15 years of age (3, $4)$.

Although carnivorous mammals (jackal, hyena, and hog), livestock (sheep, cattle, camel, and pig), and even bats can be considered as the natural rabies reservoirs, more than $95 \%$ of cases are caused by dog bites. In humans, this disease is usually caused by the bite or scratch of an infected animal. However, it can be transmitted through direct contact of saliva-contaminated materials with mucus or new skin lesions $(1,2,5)$. The documented papers on patients with the clinical symptoms of rabies with no history of a bite can confirm this transmission method (4).

After entering the peripheral nerves, the virus infects the central nervous system and multiplies in the brain. Then, through the nerves, it spreads to various tissues in the body, including the salivary glands. In humans, the onset of clinical symptoms (fever, tinnitus, buzzing, delirium, and hydrophobia) is when the rabies virus spreads throughout the body. In these cases, antiviral drugs, interferon, and high doses of anti-rabies serum do not affect preventing death (4-6).

Western Europe, Japan, Australia, and New Zealand are considered as the regions where rabies has been eliminated. However, this disease is endemic in Asian and African countries (2).

Iran, as a highly endemic country, has been affected by rabies. During 2002 - 2011, a total of 1,188,579 animal bite cases that received timely post-exposure prophylaxis were recorded in this country (7). It is documented that, on average, nine people die yearly due to rabies. There is a surveillance system to follow the registered cases. Almost 700 prevention and treatment centers are allocated for rabies in Iran (8). They should report all the rabies cases monthly to the district health center. In addition, all suspected cases referred to the hospitals are subject to immediate report by phone calls. Based on the national program for rabies control, washing the wounds, rabies immunoglobulin (if necessary), and at least three-dose vaccine injections are used for post-exposure treatment $(1,9)$.

It seems that the rabies situation is more dangerous in some parts of the country. Border provinces such as Sistan and Baluchestan can be considered as areas with local virus exchange between animals in neighboring countries and Iran (8). For example, in Zahedan, the capital of this province, the monthly frequency of animal bites in 2021 significantly increased compared to the same period in 2020 (Figure 1). On the other hand, a positive rabies test in one of the dogs that bit several people can increase the sensitivity of the issue. In 2021, the majority of animal bites occurred in urban areas (642 cases) than rural regions (197 cases), which was in contrast with national reports and some other countries $(1,2,10)$. The animals with the highest frequency were dogs (721), cats (112), foxes (2), and domestic animals (4), respectively. It seems that the lack of attention to controlling the population of stray dogs and their vaccination has led to this significant increase in the number of dog bites.

Today, at the time of the COVID-19 pandemic, the possible death due to rabies can add to the challenges of the health care system. It means that in addition to COVID-19related costs, the Iranian ministry of health should spend 
about 150 billion dollars on the purchase of rabies serum and vaccine yearly. In this regard, one of the most serious challenges in eliminating rabies is the lack of sufficient public awareness about this tragic disease in Iran (8). Similarly, a study conducted in India showed that despite the availability of effective vaccines, awareness of postexposure prevention is still poor (2). If bite victims know what to do, many of them can survive (3).

The massive killing of the dog population has been implemented for rabies control in some countries. Although controlling the stray dogs' population should be done immediately, it cannot stop a rabies outbreak without an intensive vaccination campaign for them. According to the evidence, controlling rabies through mass dog vaccination is more cost-effective than preventing human mortality (2, $11,12)$. These measures in Iran must be performed by veterinary and municipal organizations and are not within the authority of the Ministry of Health (10).

Rabies, as a fatal infectious disease, needs more attention, especially in southeastern Iran. Generally, it seems that political will and cross-sectoral coordination are the keys to improving the surveillance system for effective rabies control.

\section{Footnotes}

Authors' Contribution: M.ZB conceived the presented idea; J.N wrote the manuscript with support from M.ZB and S.S. All authors read the manuscript and confirmed the final version.

Conflict of Interests: The authors declare that they have no conflict of interest.
Funding/Support: This study received no funding.

\section{References}

1. Shirzadi MR, Pourmozafari J, Shamsipour M. Status of Animal bite and rabies cases in northeast provinces of Iran during 2009-2010. J Zoonoses. 2014;1(1).

2. Radhakrishnan S, Vanak AT, Nouvellet P, Donnelly CA. Rabies as a Public Health Concern in India-A Historical Perspective. Trop Med Infect Dis. 2020;5(4):162. doi: 10.3390/tropicalmed5040162. [PubMed: 33096767]. [PubMed Central: PMC7709690].

3. CDC. Every 9 Minutes, Someone in the World Dies of Rabies. CDC; 2015. Available from: https://www.cdc.gov/media/releases/2015/p0928rabies.html.

4. Simani S, Fayaz A, Rahimi P, Eslami N, Howeizi N, Biglari P. Six fatal cases of classical rabies virus without biting incidents, Iran 1990-2010. J Clin Virol. 2012;54(3):251-4. doi: 10.1016/j.jcv.2012.03.009. [PubMed: 22554714].

5. Shirzadi M, Gharachoulou F, Haj Rasooliha H. [Rabies vaccines WHO position paper]. Iran Ministry Health Med Educ. 2009. Persian.

6. Alizadeh L, Akbari Dana M, Barati Dowom P, Ghaemi A. Immunology of Rabies Virus in the Central Nervous System. Neurosci J Shefaye Khatam. 2015;3(3):113-20. doi: 10.18869/acadpub.shefa.3.3.113.

7. Farahtaj F, Fayaz A, Howaizi N, Biglari P, Gholami A. Human rabies in Iran. Trop Doct. 2014;44(4):226-9. doi: 10.1177/0049475514528174. [PubMed: 24662365].

8. Ebrahimzadeh Leylabadlo H, Bannazadeh Baghi H. Rabies Elimination by 2030: What Challenges Does Iran Face? Iran J Public Health. 2020;49(7):1397. doi: 10.18502/ijph.v49i7.3603.

9. Mostafavi E, Moradi GH, Rahmani KH, Jahanbakhsh F, Eybpoosh S, Keypour M, et al. Rabies surveillance system in iran: History, structures, and achievements. Iran J Epidemiol. 2020;16(1):38-47.

10. Gholami A, Fayaz A, Farahtaj F. Rabies in Iran: past, present and future. J Med Microbiol Infect Dis. 2014;2(1):1-10.

11. Windiyaningsih C, Wilde H, Meslin FX, Suroso T, Widarso HS. The rabies epidemic on Flores Island, Indonesia (1998-2003). J Med Assoc Thai. 2004;87(11):1389-93. [PubMed:15825719].

12. Abbasi M, Barfar E, Hazratian T, Abbasi R. Estimating the Cost of Prevention and Control of Rabies: A Case Study in the Northwest of Iran. Evid Based Health Policy Manag Econ. 2018;2(3):166-73. 


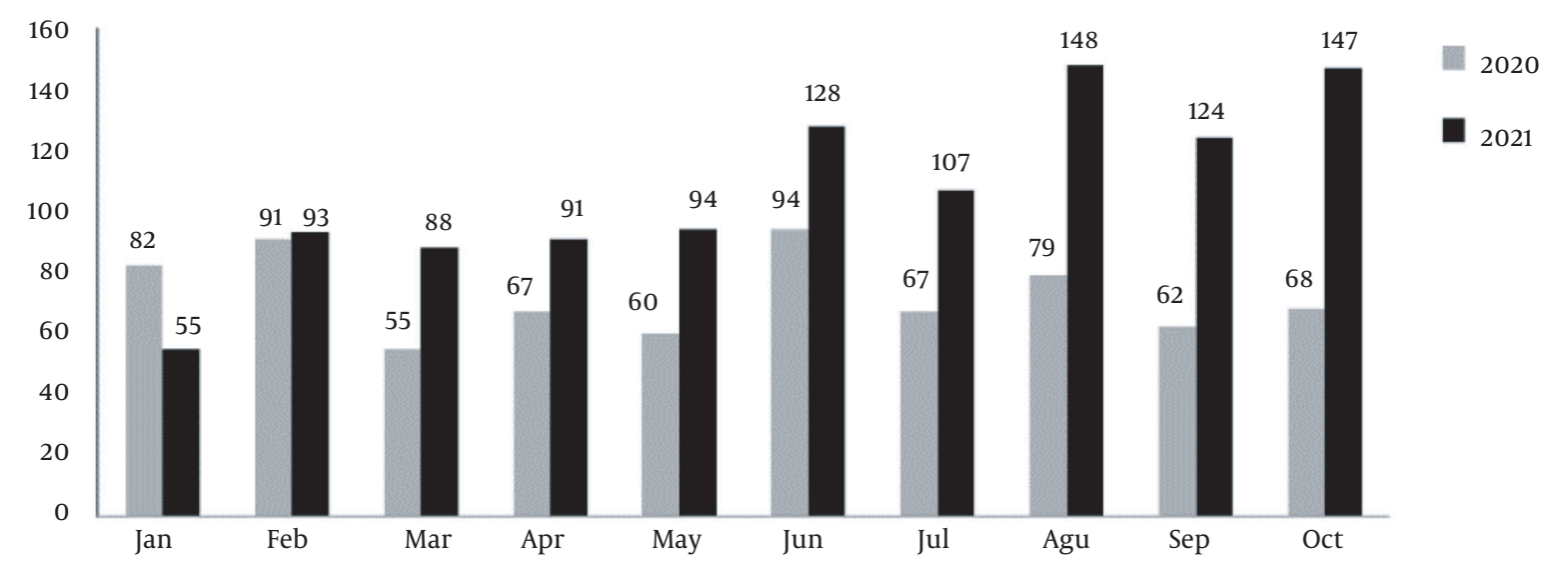

Figure 1. The monthly frequency of animal bites in Zahedan, Southeastern Iran, in 2020 - 2021 